\title{
One year into the COVID-19 pandemic - temporomandibular disorders and bruxism: What we have learned and what we can do to improve our manner of treatment
}

\author{
Alona Emodi-Perlman ${ }^{A-D}$, llana Elip-F \\ Department of Oral Rehabilitation, The Maurice and Gabriela Goldschleger School of Dental Medicine, Tel Aviv University, Israel \\ A - research concept and design; $\mathrm{B}$ - collection and/or assembly of data; $\mathrm{C}$ - data analysis and interpretation; \\ $D$ - writing the article; $E$ - critical revision of the article; $F$ - final approval of the article
}

Address for correspondence

Alona Emodi-Perlman

E-mail: dr.emodi@gmail.com

Funding sources

None declared

Conflict of interest

None declared

Received on December 25,2020

Reviewed on January 25, 2021

Accepted on January 28, 2021

Published online on May 11, 2021

Cite as

Emodi-Perlman A, Eli I. One year into the COVID-19 pandemic

- temporomandibular disorders and bruxism: What we have

learned and what we can do to improve our manner of treatment.

Dent Med Probl. 2021;58(2):215-218. doi:10.17219/dmp/132896

DOI

10.17219/dmp/132896

Copyright

○) 2021 by Wroclaw Medical University

This is an article distributed under the terms of the

Creative Commons Attribution 3.0 Unported License (CC BY 3.0)

(https://creativecommons.org/licenses/by/3.0/).

\begin{abstract}
The coronavirus disease 2019 (COVID-19) pandemic has drastically changed the routine way of life and challenged the ways in which health and dental services are provided. During the 1 st lockdown, practiced in most of the countries, routine dental procedures were suspended. Even after the lockdown was eased, visiting crowded dental clinics was still considered health-threatening, especially among populations at high risk of developing a severe reaction to COVID-19. Regretfully, in most cases, temporomandibular disorders (TMD) and bruxism were not included under the definition of emergency, leaving many patients without the possibility of consulting their dentists.

A literature search, performed about 10 months after the declaration of the pandemic, found only a few studies dealing with TMD and bruxism during COVID-19. Most of the studies indicate adverse effects on subjects' psycho-emotional status (stress, anxiety, depression), which in turn lead to the intensification of subjects' TMD and bruxism symptoms, and increased orofacial pain. Unlike other oral pathologies, which require manual interventions, chronic orofacial pain can be addressed, at least at its initial stage, through teledentistry and/or consultation.

Remote first aid for patients suffering from orofacial pain includes various kinds of treatment, such as the self-massage of tense and painful areas, stretching, thermotherapy, drug therapy, relaxation techniques, meditation, and mindfulness, all of which can be administered through the phone and/or the Internet. Relevant legal and ethical issues should be considered while using remote modes for the triage, diagnosis and treatment of chronic orofacial pain patients.
\end{abstract}

Keywords: bruxism, temporomandibular disorders, orofacial pain, COVID-19, teledentistry 


\section{Introduction}

About a year ago, the World Health Organization (WHO) was informed of cases of pneumonia of unknown cause in Wuhan, China. A novel coronavirus - severe acute respiratory syndrome coronavirus 2 (SARS-CoV-2) - was identified as the cause. ${ }^{1}$ On March $11^{\text {th }}, 2020$, due to a rapid increase in the number of cases outside China, WHO announced that the outbreak could be characterized as a pandemic. By mid-March 2020, the WHO European region had become the epicenter of the respiratory coronavirus disease 2019 (COVID-19) epidemic, reporting over $40 \%$ of the globally confirmed cases. ${ }^{1}$ At the time of this review being written, COVID-19 cases worldwide reached 78,065,178, causing the death of over $1,716,170$ people. $^{2}$

Following WHO's declaration of pandemic and due to uncertainty about the ways of spread, efficient treatment and the lack of vaccine, most countries adopted social distancing policies and partial to total lockdown. ${ }^{3}$ Routine everyday life was drastically altered and has not gone back to normal yet. This situation gave rise to severe health threats, economic uncertainty and social isolation, each of them with a potential of severe deleterious effects on both the physical and mental health of people. ${ }^{3}$

The COVID-19 pandemic has challenged the existing healthcare systems across the globe in general, and in particular, the way dental care is provided. The primary mode of spread of the virus is via respiratory droplets, which puts dentists at the spearhead of infection. ${ }^{4}$ As a result, during the $1^{\text {st }}$ lockdown, routine dental procedures were suspended in most countries. Only emergency dental procedures, specified by WHO and the American Dental Association (ADA), were authorized. ${ }^{5}$ In most countries, dentists were instructed to limit their practices to emergency and urgent dental care. ${ }^{6}$ Regretfully, temporomandibular disorders (TMD) and bruxism were not considered as such, leaving many patients without the possibility to consult their dentists in moments they might have needed them the most.

Temporomandibular disorders and bruxism are often associated with psychosocial factors, such as stress, anxiety, depression, and catastrophizing. ${ }^{7-16}$ The TMDrelated pain affects the daily activities and quality of life of numerous individuals worldwide. ${ }^{17}$

Bruxism (at daytime and/or during sleep) poses similar problems. A recent review demonstrated that patients with high levels of stress were almost 6 times more likely to report awake bruxism. Researchers explained the sustained muscle contraction as a fight-or-flight reaction and implicated that awake bruxism could be part of the defense behavior occurring in times of stress and anxiety. ${ }^{18}$

Undoubtedly, the COVID-19 pandemic has caused significant distress to billions of individuals worldwide. The most common psychosocial responses to the pandemic are stress, anxiety and depression. ${ }^{3}$

\section{Objective}

The aim of the study was to review the initial existing literature on TMD, bruxism and orofacial pain during the COVID-19 pandemic - signs and symptoms, triage, diagnosis, and management during times of psychological tension, immediate health hazards and social isolation.

\section{Method}

A data search was performed using the PubMed and Quertle databases, applying the following keywords: "COVID-19" and/or "coronavirus" in combination with “TMD”, "bruxism”, “orofacial pain”, and "teledentistry”.

The selection criteria were original research and/or review articles in the English language, published between March 1, 2020 and November 30, 2020, and relevant publications extracted from them. The final review was based on 13 items fulfilling the inclusion criteria.

\section{Results and discussion}

A cross-sectional online survey conducted in Israel and Poland during the $1^{\text {st }}$ lockdown period showed adverse effects on the psycho-emotional status of both Israeli and Polish populations, and the intensification of subjects' TMD and bruxism symptoms, leading to increased orofacial pain. ${ }^{19}$ Data arising from the COVID-19 literature supports the aggravation of signs and symptoms of TMD and bruxism among the general population and TMD patients. ${ }^{19-21}$

A survey conducted in China, which compared TMD patients to the general population and a population of orthodontic patients, concluded that during the pandemic, TMD patients exhibited higher levels of psychological distress than the other 2 populations. ${ }^{22}$ Factors such as young age, female gender, concerns about isolation, psychological barriers, and distrust were found to be associated with a high level of psychological distress and TMD pain. ${ }^{22}$ The authors suggested that their findings were due to higher levels of sympathetic activity and the release of adrenocortical steroids, leading to vasoconstriction and increased peripheral vascular resistance, triggered by the emergency and threatening situation caused by the pandemic. ${ }^{22}$ The autonomic impairment may have also led to increased sympathetic drive and the hyperarousal sensation, which create and perpetuate sleep disturbances, and this in turn may explain the aggravation of sleep bruxism. ${ }^{23}$ Additionally, a case-control study conducted among ambulatory non-hospitalized patients, who were quarantined in a designated hotel for COVID-19 patients, found that facial pain was more common among women than men. ${ }^{24}$ 
According to Almeida-Leite et al., orofacial specialists should be aware of a greater risk of developing, worsening and perpetuating TMD and bruxism (mainly awake bruxism) due to COVID-19. ${ }^{20}$ The authors suggest that guidelines for patient education, self-management, home care, and relaxation techniques, which are already available on the Web, can be useful tools in times of isolation. ${ }^{20}$

Unlike other oral pathologies, which require immediate manual interventions (i.e., caries, pulpitis, a periapical abscess, etc.), chronic orofacial pain originating from TMD and/or bruxism can be addressed, at least at its initial stage, through teledentistry and/or consultation.

Telemedicine refers to the use of information-based technologies and communication systems to deliver healthcare across distances. ${ }^{25}$ Teledentistry is not a new concept in dentistry and was used by the US military as early as in $1994 .^{26}$ Video communication was found to be feasible and effective in chronic disease management. ${ }^{27}$

Today, software such as WhatsApp, Zoom, Skype, and many others may help triage, diagnose and treat various conditions. ${ }^{28} \mathrm{Lv}$ et al. suggested video conferencing guidelines and a flow chart to help dentists in decision-making at times when face-to-face consultation is prohibited and crowded waiting rooms are health-threatening, as during COVID-19. ${ }^{28}$ Patel et al. developed an algorithm to utilize guidance from the Centers for Disease Control and Prevention (CDC), which can help decide if in-office consultation is essential or a telehealth visit is sufficient. ${ }^{29}$

As most of the instruments for the initial diagnoses of TMD and bruxism are already carried out via selfreport questionnaires, ${ }^{30-32}$ teledentistry may provide additional visual parameters, such as the verification of the range of motion, indicating painful areas, etc.

First aid for TMD patients can include conservative kinds of treatment, such as the self-massage of tense and painful areas of the masticatory muscles, which can be demonstrated via teledentistry or a link to a video demonstrating such exercises may be provided. Other techniques may include mandibular relaxation and coordination exercises, stretching, and thermotherapy. ${ }^{33}$ Drug therapy, relaxation techniques, meditation, and mindfulness can all be administered through the phone and/or the Internet, and help subjects deal with the painful condition, reduce catastrophizing and improve quality of life. ${ }^{34-36}$

One accepted mode to manage bruxism is based on increasing the patient's awareness of the syndrome (especially for awake bruxism). The methods include monitoring via the Ecological Momentary Assessment (EMA) applications for smartphones, such as the BruxApp application, ${ }^{37}$ which can help monitor the bruxing behavior and act as biofeedback. All of these can be easily administered through video communication, namely, teledentistry.

Telemedicine and teledentistry are relatively new entities, which have expanded substantiality during the pandemic. Unfamiliar legal and ethical issues may arise.
Chang et al. point out that patients should be acknowledged that, even if slight, a possibility exists that the transmitted information can be intercepted. ${ }^{38}$

Another issue, which should be addressed, is that of informed consent. As nothing replaces the gold standard evaluation of a clinical examination, informed consent should cover not only the traditional consent text, but also the possibility of the inherent risk of improper diagnosis and/or treatment. Furthermore, medico-legal, copyright, fiscal, and taxation issues should also be considered concerning the information exchange. ${ }^{39,40}$

Hopefully, the mass vaccination of the population has already started, bringing hope for a better and healthier year to come. Nevertheless, there are lessons to be learned from the pandemic. Dentists and orofacial pain specialists should adopt at least some of the new strategies, technologies and guidelines for remote treatment. This will not only ease the provision of treatment to remote populations, who lack immediate access to dental clinics, but also make us better prepared for possible future health crises. Further research should focus on providing clear guidelines for the triage, diagnosis and treatment of chronic orofacial pain, TMD and bruxism through teledentistry.

\section{ORCID iDs}

Alona Emodi-Perlman (1) https://orcid.org/0000-0001-9995-125X Ilana Eli (i) https://orcid.org/0000-0002-8601-9591

\section{References}

1. World Health Organization. Coronavirus disease (COVID-19) outbreak. https://www.euro.who.int/en/health-topics/health-emergencies/coronavirus-covid-19. Accessed November 29, 2020.

2. https://www.worldometers.info/coronavirus/. Accessed December 23, 2020.

3. Wang C, Pan R, Wan X, et al. Immediate psychological responses and associated factors during the initial stage of the 2019 coronavirus disease (COVID-19) epidemic among the general population in China. Int J Environ Res Public Health. 2020;17(5):1729. doi:10.3390/ijerph17051729.

4. Uziel N, Gilon E, Meyerson J, et al. Dental personnel in Israel, Canada, and France during the COVID-19 pandemic - attitudes, worries, emotional responses, and posttraumatic growth. Quintessence Int. 2021;0(0):444-453. doi:10.3290/j.qi.b936999

5. Kulsrud Z. World Health Organization advises delaying of nonessential dental care in areas of COVID-19 community transmission. 2020. https://www.dentistryiq.com/dentistry/article/14181510/world-healthorganization-advises-delaying-of-nonessential-dental-care-in-areasof-covid19-community-transmission. Accessed November 29, 2020.

6. Solana K. ADA develops guidance on dental emergency, nonemergency care. Recommendations part of dentists' response over COVID-19 concerns. 2020. https://www.ada.org/en/publications/ ada-news/2020-archive/march/ada-develops-guidance-on-dental-emergency-nonemergency-care. Accessed November 29, 2020.

7. Berger M, Oleszek-Listopad J, Marczak M, Szymanska J. Psychological aspects of temporomandibular disorders - literature review. Curr Issues Pharm Med Sci. 2015;28(1):55-59. doi:10.1515/cipms-2015-0044

8. Differential diagnosis and management of TMDs. In: American Academy of Orofacial Pain; de Leeuw R, Klasser GD, eds. Orofacial Pain: Guidelines for Assessment, Diagnosis, and Management. th $^{\text {th }}$ ed. Hanover Park, USA: Quintessence Publishing; 2018:143-207.

9. Lajnert V, Francisković T, Grzic R, et al. Depression, somatization and anxiety in female patients with temporomandibular disorders (TMD). Coll Antropol. 2010;34:1415-1419 
10. Wieckiewicz M, Ziętek M, Smardz J, Zenczak-Wieckiewicz D, Grychowska N. Mental status as a common factor for masticatory muscle pain: A systematic review. Front Psychol. 2017;8:646. doi:10.3389/fpsyg.2017.00646

11. Lobbezoo F, Lavigne GJ, Tanguay R, Montplaisir JY. The effect of the catecholamine precursor L-dopa on sleep bruxism: A controlled clinical trial. Mov Disord. 1997;12(1):73-78. doi:10.1002/mds.870120113

12. Manfredini D, Arreghini A, Lombardo L, et al. Assessment of anxiety and coping features in bruxers: A portable electromyographic and electrocardiographic study. J Oral Facial Pain Headache. 2016;30(3):249-254. doi:10.11607/ofph.1616

13. Manfredini D, Fabbri A, Peretta R, Guarda-Nardini L, Lobbezoo F. Influence of psychological symptoms on home-recorded sleeptime masticatory muscle activity in healthy subjects. J Oral Rehabil. 2011;38(12):902-911. doi:10.1111/j.1365-2842.2011.02226.x

14. Manfredini D, Lobbezoo F. Role of psychosocial factors in the etiology of bruxism. J Orofac Pain. 2009;23(2):153-166.

15. Pierce CJ, Chrisman K, Bennett ME, Close JM. Stress, anticipatory stress, and psychologic measures related to sleep bruxism. J Orofac Pain. 1995;9(1):51-56.

16. Winocur E, Uziel N, Lisha T, Goldsmith C, Eli I. Self-reported bruxism - associations with perceived stress, motivation for control, dental anxiety and gagging. J Oral Rehabil. 2011;38(1):3-11. doi:10.1111/ j.1365-2842.2010.02118.x

17. International Association for the Study of Pain. Temporomandibular Disorders. Orofacial Pain Fact Sheets. 2016. https://s3.amazonaws. com/rdcms-iasp/files/production/public/Content/ContentFolders/ GlobalYearAgainstPain2/20132014OrofacialPain/FactSheets/Temporomandibular_Disorders_2016.pdf. Accessed November 30, 2020.

18. Przystańska A, Jasielska A, Ziarko $M$, et al. Psychosocial predictors ofbruxism.Biomed ResInt.2019;2019:2069716.doi:10.1155/2019/2069716

19. Emodi-Perlman A, Eli I, Smardz J, et al. Temporomandibular disorders and bruxism outbreak as a possible factor of orofacial pain worsening during the COVID-19 pandemic - concomitant research in two countries. J Clin Med. 2020;9(10):3250. doi:10.3390/jcm9103250

20. Almeida-Leite CM, Stuginski-Barbosa J, Rodrigues Conti PC. How psychosocial and economic impacts of COVID-19 pandemic can interfere on bruxism and temporomandibular disorders? J Appl Oral Sci. 2020;28:e20200263. doi:10.1590/1678-7757-2020-0263

21. Ignacio de Oliveira SS, Melo Gonçalvez SL, de Mello Weig K, et al. Temporomandibular disorders: Guidelines and self-care for patients during COVID-19 pandemic. Braz Dent Sci. 2020;23(2):Suppl 2. doi.org/10.14295/bds.2020.v23i2.2255

22. Wu Y, Xiong $X$, Fang $X$, et al. Differences of psychological status of TMD patients, orthodontic patients and the general population during the COVID-19 epidemic: A cross-sectional study. Res Sq preprint. 2020. doi:10.21203/rs.3.rs-18915/v1

23. Liu BCL, Lee IC, Lo LJ, Ko EWC. Investigate the oral health impact and quality of life on patients with malocclusion of different treatment needs. Biomed J. 2019;42(6):422-429. doi:10.1016/j.bj.2019.05.009

24. Biadsee A, Biadsee A, Kassem F, Dagan O, Masarwa S, Ormianer Z. Olfactory and oral manifestations of COVID-19: Sex-related symptoms - a potential pathway to early diagnosis. Otolaryngol Head Neck Surg. 2020;163(4):722-728. doi:10.1177/0194599820934380

25. Zimlichman E. Telemedicine: Why the delay? Isr Med Assoc J. 2005;7(8):525-526.

26. Rocca MA, Kudryk VL, Pajak JC, Morris T. The evolution of a teledentistry system within the Department of Defense. Proc AMIA Symp. 1999;921-924

27. Mallow JA, Petitte T, Narsavage G, et al. The use of video conferencing for persons with chronic conditions: A systematic review. Ehealth Telecommun Syst Netw. 2016;5(2):39-56. doi:10.4236/etsn.2016.52005

28. Lv N, Sun M, Polonowita A, Mei L, Guan G. Management of oral medicine emergencies during COVID-19: A study to develop practise guidelines. J Dent Sci. 2021;16(1):493-500. doi:10.1016/j.jds.2020.07.016

29. Patel IY, Patel SI, Parthasarathy S, Sheats RD. Reopening an academic dental sleep medicine and orofacial pain clinic amid COVID-19. J Dent Sleep Med. 2020;7(3). doi:10.15331/jdsm.7140

30. Lövgren A, Marklund S, Visscher CM, Lobbezoo F, HäggmanHenrikson B, Wänman A. Outcome of three screening questions for temporomandibular disorders (3Q/TMD) on clinical decisionmaking. J Oral Rehabil. 2017;44(8):573-579. doi:10.1111/joor.12518
31. Rodrigues-Bigaton D, de Castro EM, Pires PF. Factor and Rasch analysis of the Fonseca anamnestic index for the diagnosis of myogenous temporomandibular disorder. Braz J Phys Ther. 2017;21(2):120-126. doi:10.1016/j.bjpt.2017.03.007

32. Schiffman E, Ohrbach $R$, Truelove $E$, et al. Diagnostic criteria for temporomandibular disorders (DC/TMD) for clinical and research applications: Recommendations of the International RDC/TMD Consortium Network and Orofacial Pain Special Interest Group. J Oral Facial Pain Headache. 2014;28(1):6-27. doi:10.11607/jop.1151

33. Harper DE, Schrepf A, Clauw DJ. Pain mechanisms and centralized pain in temporomandibular disorders. J Dent Res. 2016;95(10):1102-1108. doi:10.1177/0022034516657070

34. Crowley C, Kapitula LR, Munk D. Mindfulness, happiness, and anxiety in a sample of college students before and after taking a meditation course. J Am Coll Health. 2020. doi:10.1080/07448481.2020.1754839

35. Gu Q, Hou JC, Fang XM. Mindfulness meditation for primary headache pain: A meta-analysis. Chin Med J (Engl). 2018;131(7):829-838. doi:10.4103/0366-6999.228242

36. Behan $C$. The benefits of meditation and mindfulness practices during times of crisis such as COVID-19. Ir J Psychol Med. 2020:1-3. doi:10.1017/ipm.2020.38

37. Manfredini D, Bracci A, Djukic G. BruxApp: The ecological momentary assessment of awake bruxism. Minerva Stomatol. 2016;65(4):252-255.

38. Chang SW, Plotkin DR, Mulligan R, Polido JC, Mah JK, Meara JG. Teledentistry in rural California: A USC initiative. J CalifDent Assoc. 2003;31(8):601-608 .

39. Wallace G. Information technology and telemedicine. CMAJ. 2001;165(6):777-779.

40. Jampani ND, Nutalapati R, Dontula BSK, Boyapati R. Applications of teledentistry: A literature review and update. J Int Soc Prev Community Dent. 2011;1(2):37-44. doi:10.4103/2231-0762.97695 